\title{
Perspectives on Carbon Nanotube-Based Scaffolds in Nerve Tissue Engineering
}

\section{Shang-Tian Yang* and Ru Zang}

Department of Chemical and Biomolecular Engineering, The Ohio State University, Columbus, Ohio, USA

To improve the health and quality of life of patients suffering from neural degeneration diseases or brain and spinal cord injuries, much research has been dedicated to the repairing and regeneration of neural tissues. Although allogenic grafts have no supply limitation, they often cause undesirable immune responses. Thus, autologous grafts are usually used to treat neural defects. However, the short life of nerves and a mismatch of nerve cable dimension between the donor graft and the receptor nerve limit its clinical applications [1]. Nerve tissue engineering has emerged as a highly promising alternative strategy to neural therapy, aimed at rebuilding the lesioned circuits of the central and peripheral nervous systems, while minimizing body's immune responses with engineered nerves [2].

One focus in tissue engineering is the development and design of extracellular scaffolds that mimic natural extracellular matrix (ECM) to support three-dimensional (3-D) cell proliferation and tissue regeneration. A natural tissue scaffold comprises of nanofibrous materials that provide a nano-textured surface supporting cell adhesion, migration, signaling, and morphogenesis. In vivo, cells are surrounded by ECM that is characterized by a natural web of hierarchically organized nanofibers that play a vital role in directing cellular behaviors via cellsurface interactions [3]. Cell-surface interactions can be controlled or regulated by varying the surface chemistry, mechanical properties, and topography. For nerve tissue engineering, nanomaterials have been increasingly used in developing nano-engineered scaffolds to regulate cell-surface interactions and promote neural regeneration following injury.

Carbon nanotubes (CNTs), which were first discovered in 1991 by Sumio Iijima [4], have been at the forefront of nanotechnology, because their unique physicochemical properties enable the development of a variety of miniaturized devices with remarkable performance. Recently, CNTs have also gained great attention for their potential as tissue engineering scaffolds. Carbon nanotubes, when used in nerve tissue engineering, exhibit many stimulating effects, including reestablishing the intricate connections between neurons, and guiding and enhancing the intrinsic capacity of the brain to reorganize in a controlled fashion via regulating the interactions between scaffolds and biological cell membranes [2]. The dimension, diameter and length of CNTs are analogous to those of the natural ECM proteins, including laminin and collagen [5]. The size, high electrical conductivity and aspect ratio, and large surface area of CNTs favor their interactions with distal dendrites, which promote nerve regeneration [2]. Also, CNT-based scaffolds can maintain structural integrity as they have similar mechanical properties to structural proteins found in natural ECM [5]. Recent research has shown that CNTs could aid nerve tissue regeneration and deliver drugs to repair damaged neurons associated with disorders such as epilepsy, Parkinson's disease and even paralysis [6]. Moreover, the incorporation of CNTs in scaffolds provides electrical conductivity [78], which may aid in directing cell growth. Several recent studies have shown that the use of an electrical field would aid bone regeneration, neural regeneration and wound healing [9-11]. Therefore, CNTs as nanofibrous scaffolds hold promises for enhancing the restoration of lost nerve functions.
In addition to electrical conductivity, the ideal scaffold for nerve regeneration should also possess appropriate magnetic properties. Magnetic fields have profound effects on numerous biological processes. Pulse magnetic fields (PMFs) were found to stimulate nerve growth, regeneration, and functional recovery of peripheral nerves in both in vitro and in vivo studies [12]. Recent studies also showed that mechanical tension caused by a magnetic field played a role in stimulating axon growth in vitro and in vivo. For example, mechanical tension created by using super-paramagnetic nanoparticles in a magnetic field stimulated neurites outgrowth or axon elongation of neurons in the central nervous systems [13]. Therefore, scaffolds that can be manipulated in situ using magnetic fields according to the individual patient's needs should have clinical applications, and thus are desirable.

In summary, biologically compatible scaffolds should have 3-D structures with nano-features mimicking microenvironments found in native extracellular matrices. For nerve tissue regeneration, they should also possess appropriate conductive and magnetic properties, which can be afforded by carbon nanotubes and magnetic nanoparticles, respectively. These next-generation tissue scaffolds can promote nerve cell proliferation and serve as a permissive bridge for nerve regeneration. With the development of these novel scaffolds for tissue regeneration, there is now considerable optimism among neurologists that effective clinical therapies are within reach.

\section{References}

1. Subramanian A, Krisshnan UM, Stethuranman (2009) Development of biomaterial scaffold for nerve regeneration for nerve tissue engineering: Biomaterial mediated neural regeneration. J Biomed Sci 16: 108.

2. Cellot G, Toma FM, Varley ZK, Laishram J, Villari A, et al. (2011) Carbon nanotubes scaffolds tune synaptic strength in cultured neural circuits: Novel frontiers in nanomaterial-tissue interactions. J Neurosci 31: 12945-12953.

3. Shi J, Votruba AR, Farokhzad OC, Langer R (2010) Nanotechnology in drug discovery and tissue engineering: From discovery to applications. Nano Lett 10: 3223-3230.

4. lijima S (1991) Helical microtubules of graphitic carbon. Nature 354: 56-58.

5. Abbott A (2003) Cell culture: biology's new dimension. Nature 424: 870-872.

6. Kelly B (2009) Small concerns: nanotech regulations and risk management. SPIE Newsroom.

*Corresponding author: Shang-Tian Yang, Professor, Department of Chemica and Biomolecular Engineering, The Ohio State University, 140 West 19th Avenue Columbus, Ohio 43210, USA, Tel: (614) 292-6611; Fax: (614) 292-3769; E-mail: yangst.ohio.state.university@gmail.com

Received December 14, 2011; Accepted December 20, 2011; Published December 22, 2011

Citation: Yang S, Zang R (2012) Perspectives on Carbon Nanotube-Based Scaffolds in Nerve Tissue Engineering. J Tissue Sci Eng 3:e108. doi:10.4172/21577552.1000e108

Copyright: (c) 2012 Yang S, et al. This is an open-access article distributed under the terms of the Creative Commons Attribution License, which permits unrestricted use, distribution, and reproduction in any medium, provided the original author and source are credited. 
Citation: Yang S, Zang R (2012) Perspectives on Carbon Nanotube-Based Scaffolds in Nerve Tissue Engineering. J Tissue Sci Eng 3:e108. doi:10.4172/2157-7552.1000e108

Page 2 of 2

7. MacDonald RA, Voge CM, Kariolis M, Stegemann JP (2008) Carbon nanotubes increase the electrical conductivity of fibroblast-seeded collagen hydrogels. Acta Biomater 4: 1583-1592.

8. Edwards SL, Church JS, Werkmeister JA, Ramshaw JA (2009) Tubular microscale multiwalled carbon nanotube-based scaffolds for tissue engineering. Biomaterials 30: 1725-1731.

9. Wan AC, Ying JY (2010) Nanomaterials for in situ cell delivery and tissue regeneration. Adv Drug Deliv Rev 62: 731-740.

10. Shi X, Sitharaman B, Pham QP, Liang F, Wu K, et al. (2007) Fabrication of porous ultra-short single-walled carbon nanotube nanocomposite scaffolds for bone tissue engineering. Biomaterials 28: 4078-4090.

11. Edwards SL, Werkmeister JA, Ramshaw JA (2009) Carbon nanotubes in scaffolds for tissue engineering. Expert Rev Med Devices 6: 499-505.

12. Gunay I, Mert T (2011) Pulsed magnetic fields enhance the rate of recovery of damaged nerve excitability. Bioelectromagnetics 32: 200-208.

13. Silva MN, Almeida MV, Goldberg JL (2007) Developing super-paramagnetic nanoparticles for central nervous system axon regeneration. Nanotech 2: 791 794. 